\title{
LONG-TERM SURVIVAL BENEFITS OF CORONARY ARTERY BYPASS GRAFTING AND PERCUTANEOUS TRANSLUMINAL ANGIOPLASTY IN PATIENTS WITH CORONARY ARTERY DISEASE
}

Robert $\mathrm{H}$. Jones, $\mathrm{MD}^{\mathrm{a}}$

Karen Kesler, MS ${ }^{b s}$

Harry R. Phillips III, MD ${ }^{\text {b§ }}$

Daniel B. Mark, MD ${ }^{\text {b§ }}$

Peter K. Smith, MD

Charlotte L. Nelson, MS ${ }^{\text {b\$ }}$

Mark F. Newman, MD ${ }^{\text {cs }}$

Joseph G. Reves, MD ${ }^{\text {c§ }}$

Robert W. Anderson, $\mathrm{MD}^{\mathrm{a}}$

Robert M. Califf, $\mathrm{MD}^{\mathrm{b} s}$
The purpose of this study was to evaluate long-term survival benefits of bypass surgery and angioplasty versus medical therapy in 9263 patients at Duke University Medical Center between 1984 and 1990 with coronary artery disease confirmed by cardiac catheterization to involve one, two, or three vessels. Clinical data were prospectively entered into an established cardiovascular database, and annual follow-up was $97 \%$ complete for a mean interval of 5.3 years and a maximal interval of 10 years. Outcomes were analyzed with the Coronary Artery Surgery Study "method A" to define patient groups treated by medicine $(n=2449)$, angioplasty $(n=$ 2924), or bypass surgery $(n=3890$ ). Differences among treatment groups in baseline characteristics were adjusted by Cox proportional hazard models. The anatomic severity of coronary artery stenosis best defined survival benefit from bypass surgery and angioplasty versus medical treatment. One or both interventional treatments provided better long-term survival than did medical treatment for all levels of disease severity. All patients with single-vessel disease, except those with at least $95 \%$ proximal left anterior descending stenosis, benefited from angioplasty versus bypass. All patients with three-vessel disease and those two-vessel patients with $\mathbf{2 9 5 \%}$ proximal left anterior descending stenosis benefited from bypass surgery versus angioplasty. All other patients with two-vessel disease and those with $\geq 95 \%$ proximal left anterior descending stenosis only had similar survival with either interventional treatment. The absolute survival benefit was greatest for patients with severe three-vessel disease treated with bypass surgery. (J Thorac Cardiovasc Surg 1996;111:1013-25)
Cosions oronary artery bypass grafting (CABG) has been used with increased frequency since its introduction into clinical practice more than 25 years ago. ${ }^{1}$ Dramatic increases in the use of percutaneous transluminal coronary angioplasty (PTCA) during the past

From the Heart Center and the Departments of Surgery, ${ }^{a}$ Medicine, ${ }^{\mathrm{b}}$ and Anesthesiology, ${ }^{\mathrm{c}}$ Duke University Medical Center, Durham, N.C.

Supported in part by grants PORT I/AHCPR HS06503 and AHCPR HS05635.

Read at the Seventy-fifth Annual Meeting of The American Association for Thoracic Surgery, Boston, Mass., April 23-26, 1995.

Received for publication April 27, 1995; revisions requested June 13, 1995; revisions received Dec. 7, 1995; accepted for publication Dec. 18, 1995.

Address for reprints: Robert H. Jones, MD, P.O. Box 2986, Duke University Medical Center, Durham, NC 27710.

$s_{\text {By invitation. }}$

Copyright (C) 1996 by Mosby-Year Book, Inc.

$0022-5223 / 96 \$ 5.00+0 \quad \mathbf{1 2 / 6 / 7 1 2 5 8}$
15 years have modified the characteristics of populations of patients treated by CABG. ${ }^{2}$ The effects of this major shift in practice on survival of patients with coronary artery disease (CAD) remains unknown. We previously reported intermediate-term survival for 9263 patients with CAD confirmed by coronary angiography at Duke University Medical Center between March 1984 and August 1990. ${ }^{3}$ Detailed description of baseline characteristics and prospective follow-up of the entire patient cohort provide unique comparative data. We now report long-term survival for as long as 10 years on this patient cohort to establish the relative and absolute values of PTCA, CABG, and medical treatment and as a general framework of information to aid treatment selection for individual patients with CAD.

\section{Methods}

Since 1971, clinical and coronary angiography data have been prospectively entered into a database on patients 


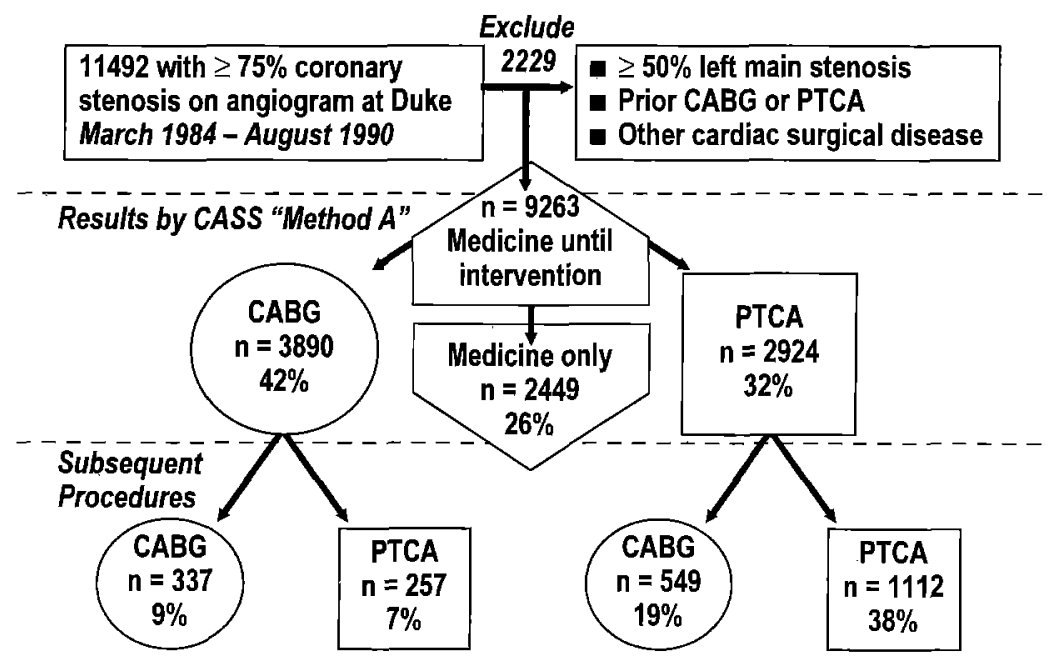

Fig. 1. Schematic diagram summarizes selection of patients for study, mode of assignment to treatment groups, and performance of subsequent procedures.

catheterized at Duke University Medical Center. Between March 1984 and August 1990, 11,492 patients were found by angiography to have at least $75 \%$ stenosis in one or more coronary arteries. To select only those patients who might be reasonable candidates for either primary $\mathrm{CABG}$ or PTCA, 2229 patients were excluded because of at least $50 \%$ left main stenosis $(n=691)$, previous CABG $(n=$ $527)$ or PTCA $(n=253)$, or $3+$ to $4+$ mitral regurgitation $(n=422)$. The Coronary Artery Surgery Study (CASS) "method A" of assignment of patients to treatment subgroups was used. ${ }^{4-6}$ All patients were initially assigned to the medical subgroup and remained in this group unless CABG or PTCA was performed. For patients undergoing revascularization procedures, medical follow-up was censored at the time of the procedure and follow-up was restarted at a new zero time in the appropriate revascularization group. Once a patient was assigned to a revascularization group, all subsequent outcome events were assigned to that treatment group regardless of subsequent therapeutic crossovers.

This approach to patient subgrouping resulted in placement of 9263 patients initially in the medical subgroup, 2449 of whom remained on medical treatment throughout the study (Fig. 1). CABG was performed in 3890 patients $(42 \%)$, and 3080 of these patients $(79 \%)$ underwent their initial procedure within 60 days of cardiac catheterization. In the CABG group, 337 patients (9\%) underwent subsequent $\mathrm{CABG}$ and $257(7 \%)$ patients underwent subsequent PTCA. PTCA was the initial treatment for 2924 (32\%) of the 9263 patients, and 2626 of these patients $(90 \%)$ underwent their initial procedure within 60 days of cardiac catheterization. In the PTCA group, 549 patients (19\%) underwent subsequent CABG and 1112 patients $(38 \%)$ underwent subsequent PTCA. Subsequent procedures were more common and occurred earlier in patients treated initially with PTCA versus CABG (Fig. 2). Including both initial and secondary procedures, $\mathrm{CABG}$ was performed in about one half of the 9263 patients and
PTCA was performed in about one third at some time during the 10-year study interval.

Baseline clinical and cardiac catheterization characteristics of the three treatment populations did not change substantially from those described previously. ${ }^{3}$ Performance of $468 \mathrm{CABG}$ and 136 PTCA procedures removed 609 patients from the medical group in the interval since intermediate-term results were reported. The revascularization techniques used have been described previously, and early mortality rates associated with more recent PTCA and CABG procedures were similar to those previously described.

The extent of CAD has long been known to be a principal determinant of the survival benefit of revascularization. This study used a previously described anatomic CAD index derived from a group of 6034 patients with medically treated CAD seen at Duke University Medical Center from 1969 to $1984 .^{7}$ The relative prognostic weight of this $\mathrm{CAD}$ severity score was determined by the numbers of stenoses of at least $75 \%$ and at least $95 \%$, as well as location and severity of stenosis in the left anterior descending (LAD) coronary artery. This CAD score was scaled so that zero was the risk of cardiac death in patients with no CAD and 100 was the risk of cardiac death in patients with at least 95\% stenosis of the left main coronary artery. The full CAD severity score has 12 levels. In this report, exclusion of patients with less than $75 \%$ stenosis and those with left main disease resulted in nine coronary anatomy groups representing a continuum of one-, two-, and three-vessel CAD (Fig. 3).

Patient follow-up data at 6 months and every year on the anniversaty of initial presentation, obtained by means of self-administered questionnaires and telephone contact, were $97 \%$ complete. Deaths were classified by an independent mortality committee into cardiovascular and noncardiovascular causes. The primary endpoint for this study was cardiovascular death. 


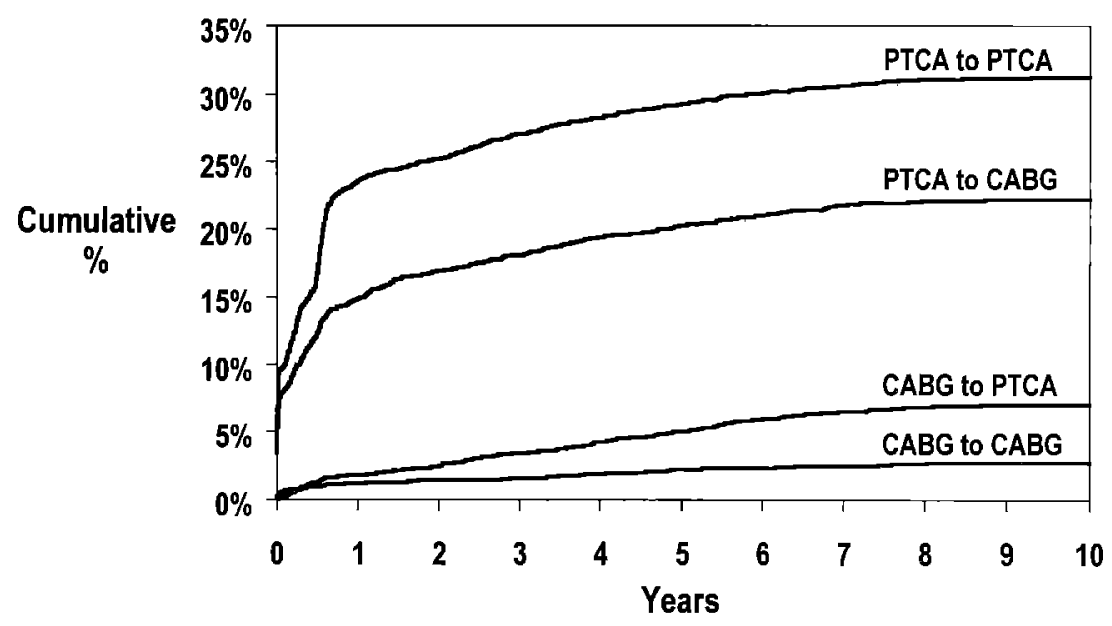

Fig. 2. Cumulative percentage of patients undergoing a secondary procedure during the 10-year study period for patients initially treated with PTCA or CABG.

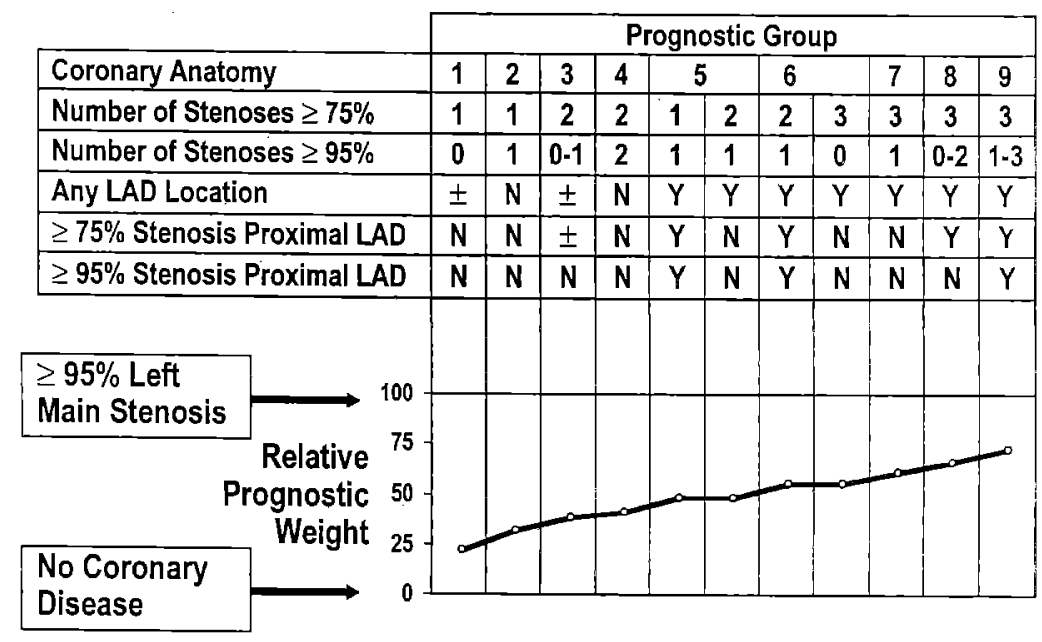

Fig. 3. Coronary anatomy score was derived for 6034 medically treated patients undergoing coronary angiography at Duke University Medical Center between 1969 and 1984. The anatomic classification of coronary artery stenosis is related to the corresponding relative prognostic weight.

Statistical analysis. A Cox proportional hazards regression model stratified by treatment group was used to adjust for baseline differences. An additional model including treatment as a covariate was used to obtain hazard ratios. These are presented with $99 \%$ confidence limits (CL) to compare the relative benefits of each of the three possible pairs of the three treatments for each of the coronary artery subgroups. Adjusted Kaplan-Meier survival curves were constructed for each coronary anatomy group and each treatment. The time course of differences in survival illustrates the absolute survival differences among the three treatments. These data are presented as the number of extra patients surviving for each 100 patients receiving the more beneficial treatment versus the treatment with lesser benefit.

\section{Results}

The mean follow-up interval for the 9263 patients after assignment to a treatment group was 5.3 years (20th, 50th, and 75th percentiles 3.9, 5.5, and 7.1 years, respectively). At some time during the 10 -year study, cardiovascular death occurred in 541 (12\%) of the 3890 CABG-treated patients, 328 (9\%) of the 2924 of the PTCA-treated patients, and 796 (9\% of $9263,33 \%$ of 2449) of the patients treated medically. Separate Cox proportional hazards models in the three treatment subgroups identified only nine clinical and angiographic characteristics related to cardiac death. The relative importance of seven of 


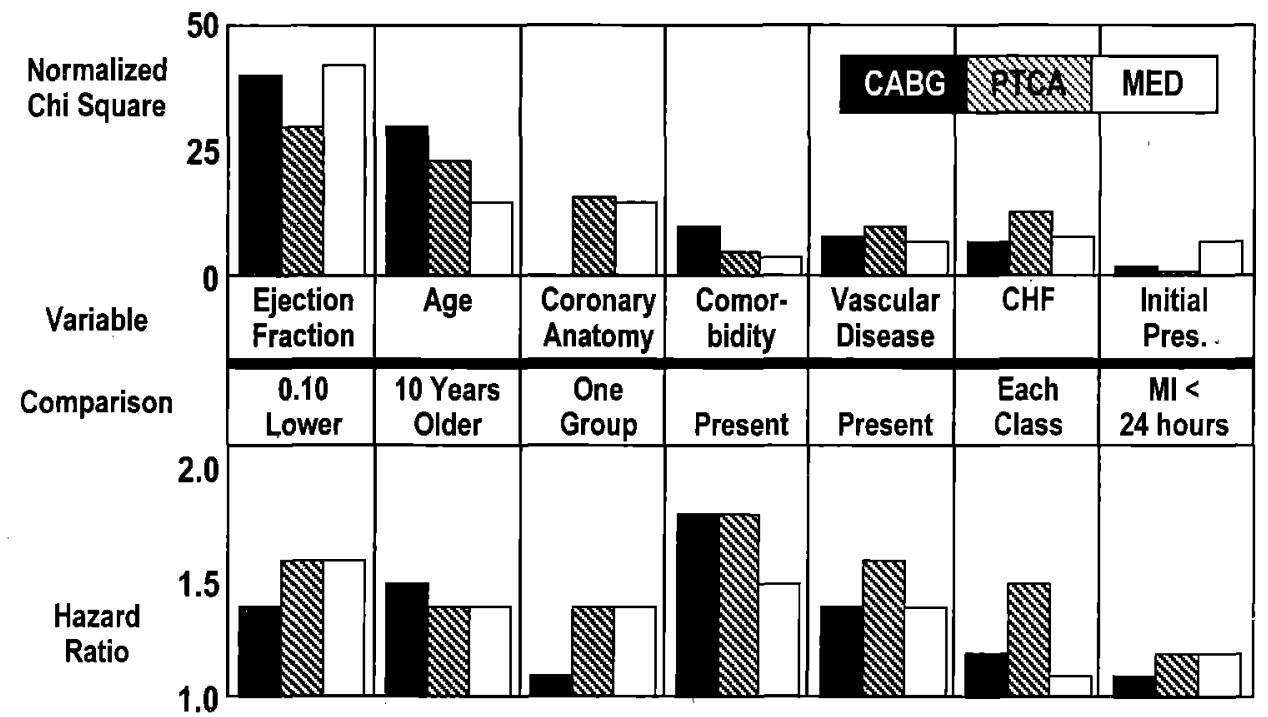

Fig. 4. The relative importance of seven most important baseline characteristics for predicting cardiac death for patients within the three treatment groups is summarized by the normalized $\chi^{2}$ values of Cox models. The Cox model $\chi^{2}$ is influenced both by the relative biologic importance of the variable and its prevalence in a population. The hazard ratio of each variable shows the relative increase in mortality versus the treatment group average that would be expected for an individual patient if the variable is present at the specified level. $M E D$, Medical treatment; $C H F$, congestive heart failure; $M I$, myocardial infarction.
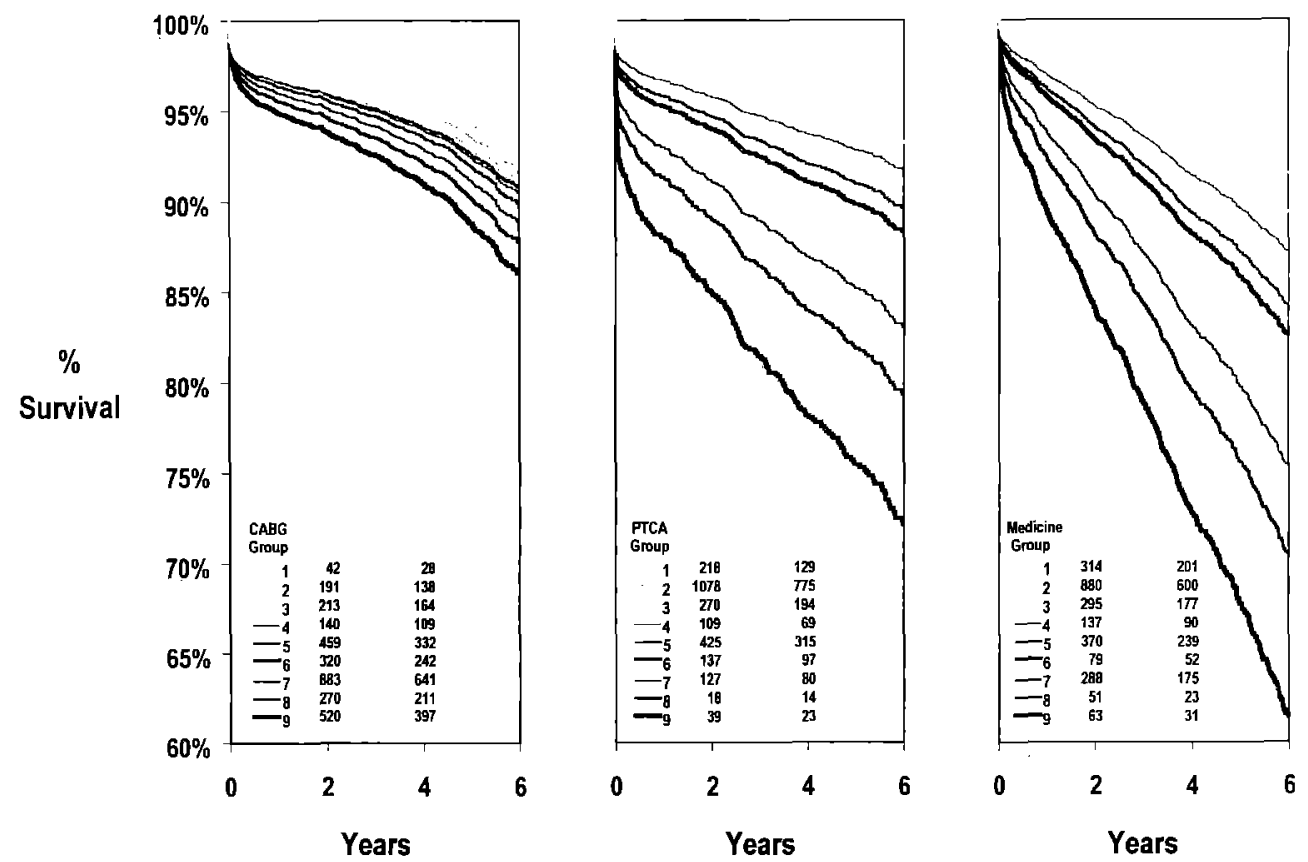

Fig. 5. Adjusted Kaplan-Meier curves for the nine anatomic CAD groups show the time courses of cardiac death in the three treatment groups. The numbers of patients in each of the three treatment groups are tabulated at 2 and 4 years. 
Table I. Five-year survival by treatment in each anatomic subgroup

\begin{tabular}{|c|c|c|c|c|c|c|c|c|c|}
\hline \multirow{2}{*}{$\begin{array}{c}\text { Coronary } \\
\text { anatomy } \\
\text { group }\end{array}$} & \multirow{2}{*}{$\begin{array}{c}\text { Diseased } \\
\text { vessels }\end{array}$} & \multirow[b]{2}{*}{$\geq 95 \%$} & \multirow[b]{2}{*}{$L A D$} & \multicolumn{2}{|c|}{$C A B G$} & \multicolumn{2}{|c|}{$P T C A$} & \multicolumn{2}{|c|}{ Medicine } \\
\hline & & & & Observed & Adjusted & Observed & Adjusted & Observed & Adjusted \\
\hline 1 & 1 & No & & 0.97 & 0.92 & 0.95 & 0.96 & 0.94 & 0.93 \\
\hline 2 & 1 & Yes & & 0.89 & 0.91 & 0.93 & 0.94 & 0.93 & 0.91 \\
\hline 3 & 2 & No & & 0.92 & 0.90 & 0.91 & 0.92 & 0.91 & 0.88 \\
\hline 4 & 2 & Yes & & 0.81 & 0.90 & 0.87 & 0.90 & 0.91 & 0.86 \\
\hline 5 & $\begin{array}{l}1 \\
2\end{array}$ & $\begin{array}{l}\text { Yes } \\
\text { Yes }\end{array}$ & $\begin{array}{l}\geq 95 \% \text { Proximal } \\
\geq 95 \%\end{array}$ & 0.86 & 0.89 & 0.85 & 0.88 & 0.90 & 0.83 \\
\hline 6 & $\begin{array}{l}2 \\
3\end{array}$ & $\begin{array}{l}\text { Yes } \\
\text { No }\end{array}$ & $\begin{array}{l}\geq 95 \% \text { Proximal } \\
\text { Yes }\end{array}$ & 0.87 & 0.89 & 0.80 & 0.85 & 0.90 & 0.79 \\
\hline 7 & 3 & Yes & Yes & 0.85 & 0.88 & 0.74 & 0.80 & 0.89 & 0.73 \\
\hline 8 & 3 & Yes & Proximal & 0.81 & 0.86 & 0.83 & 0.75 & 0.88 & 0.67 \\
\hline 9 & 3 & Yes & $\geq 95 \%$ Proximal & 0.83 & 0.85 & 0.61 & 0.68 & 0.85 & 0.59 \\
\hline
\end{tabular}

the nine most dominant variables for predicting cardiac death in all three treatment groups is depicted by the relative $\chi^{2}$ (Fig. 4). Corresponding hazard ratios relate to the relative increase in risk of cardiac death for an individual patient imposed by presence of these risk markers.

The Cox proportional hazards model incorporating all significant variables adjusted observed survival for differences in clinical and angiographic characteristics among the three treatment groups. The resulting adjusted Kaplan-Meier 6-year survival curves for patients in the three treatment groups reflect the expected survival of an average patient in each of the nine anatomic subgroups (Fig. 5). Numeric values for 5-year observed and adjusted survival are summarized in Table I. The negative influence of anatomic disease severity on survival is apparent in all three treatment groups, with mortality consistently increasing as a function of increasing anatomic subgroup number. In addition, the greatest differences among treatment groups are apparent in those with the most severe disease.

Patient and practitioner choices of treatment were influenced by specific characteristics in individual cases (Fig. 6). General knowledge of the benefits of specific treatment modalities for differing coronary anatomies appears to have guided treatment selection. Treatment choice for an individual patient that would not be usual for the average of the group therefore, might suggest unusual clinical features that might not be well described in models of data for the entire population. Moreover, statistical certainty remains influenced by the number of events in each treatment subgroup, and statements of treatment benefit are weakest in treatment subgroups containing small numbers of patients. Although

\section{Treatment Received}

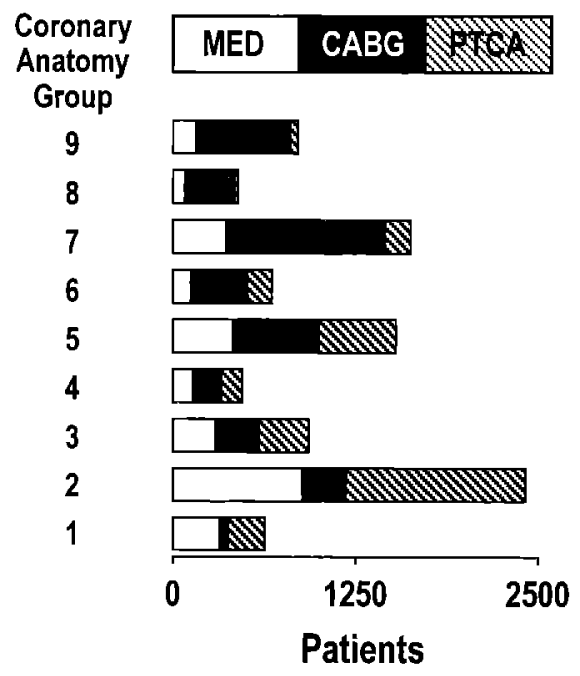

Fig. 6. Data reflect actual treatment numbers of patients included in this study in each of nine anatomic subgroups who received $\mathrm{CABG}$, PTCA, or medical treatment (MED).

statistical models can be used to adjust for differences in baseline characteristics within anatomic subgroups, the frequency of use of each of the three treatments within the nine anatomic subgroups must be considered in interpreting comparative data.

Hazard ratios were calculated from adjusted survival in each anatomic subgroup to depict the relative benefits of the three possible pairs of treatments. Hazard ratios comparing CABG and medical treatment demonstrate groups 1 through 4 to have no significant difference, but the trend in these groups favors medicine over $\mathrm{CABG}$ for treatment of 


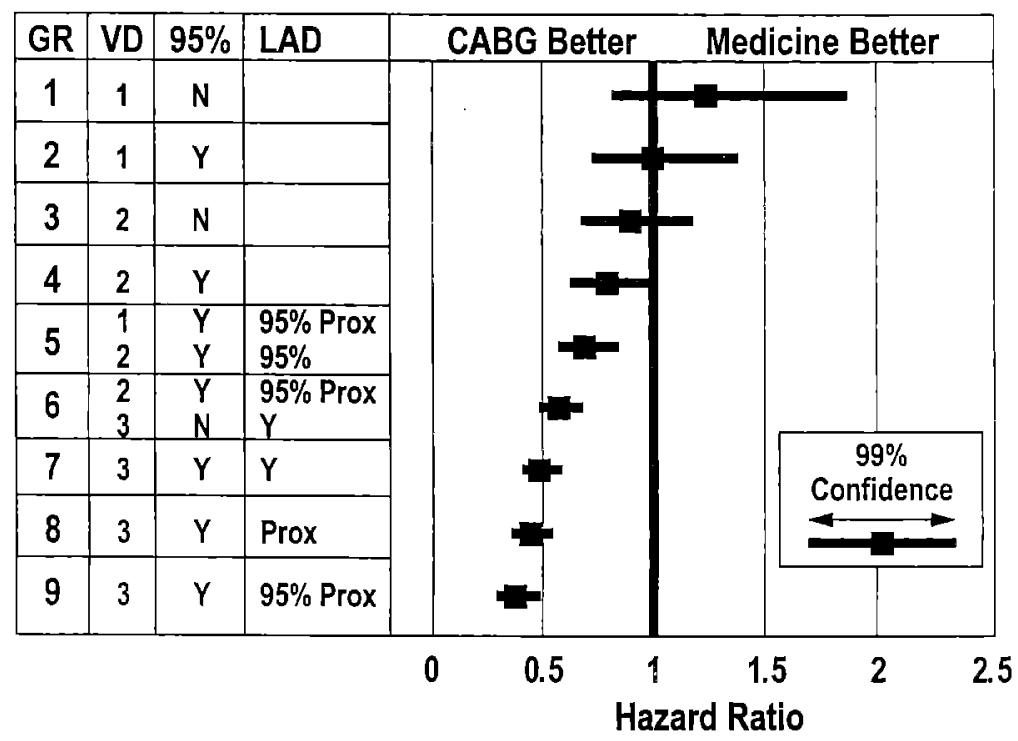

Fig. 7. Adjusted hazard ratios comparing $\mathrm{CABG}$ and medicine for the nine coronary anatomy groups. VD, Number of diseased vessels; $95 \%, \geq 95 \%$ coronary artery stenosis; Prox, proximal.

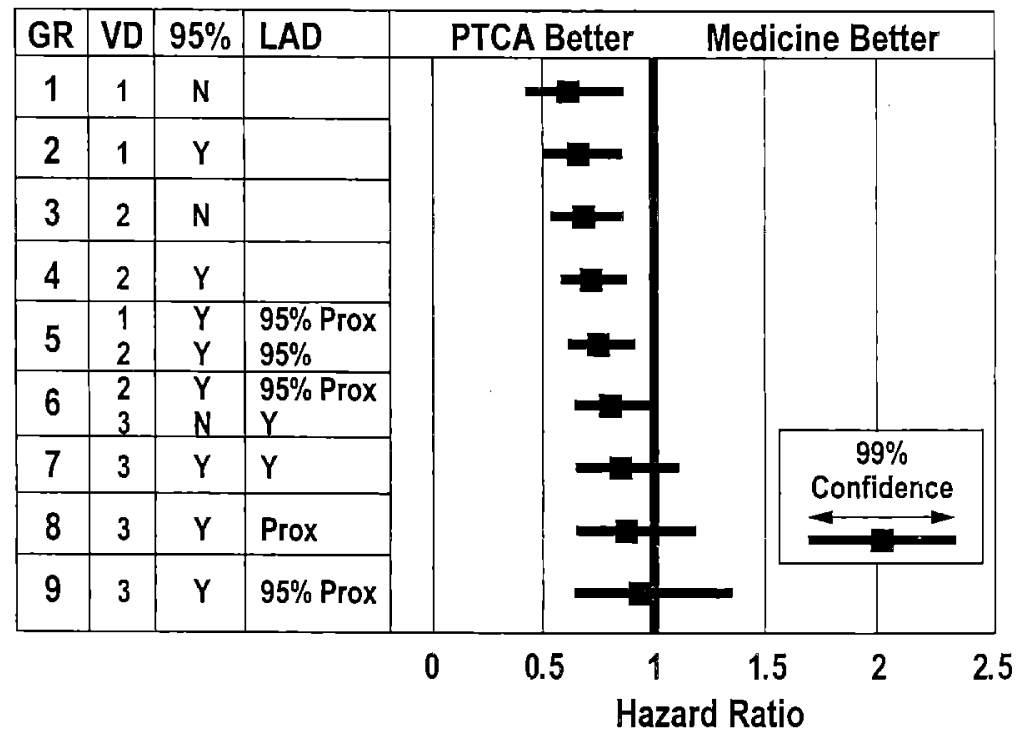

Fig. 8. Adjusted hazard ratios comparing PTCA and medicine for the nine coronary anatomy groups. VD, Number of diseased vessels; $95 \%$, $\geq 95 \%$ coronary artery stenosis; Prox, proximal.

patients with the least severe CAD (Fig. 7). CABG improves survival relative to medical treatment in subgroups 5 through 9, and the benefit increases progressively with increasing CAD severity. Adjusted hazard ratios comparing PTCA and medicine show anatomic groups 1 through 5 to have a definite survival benefit with PTCA (Fig. 8). The relative benefit trend among groups is the opposite of that of CABG versus medicine. Adjusted hazard ratios comparing relative benefit of CABG and PTCA show unequivocal benefit of PTCA in groups 1 and 2 (Fig. 9). The mean differences in groups 3 and 4 suggest a benefit for PTCA, but this difference does not reach statistical significance. Group 5 shows equivalent outcomes for both interventional procedures. Pa- 


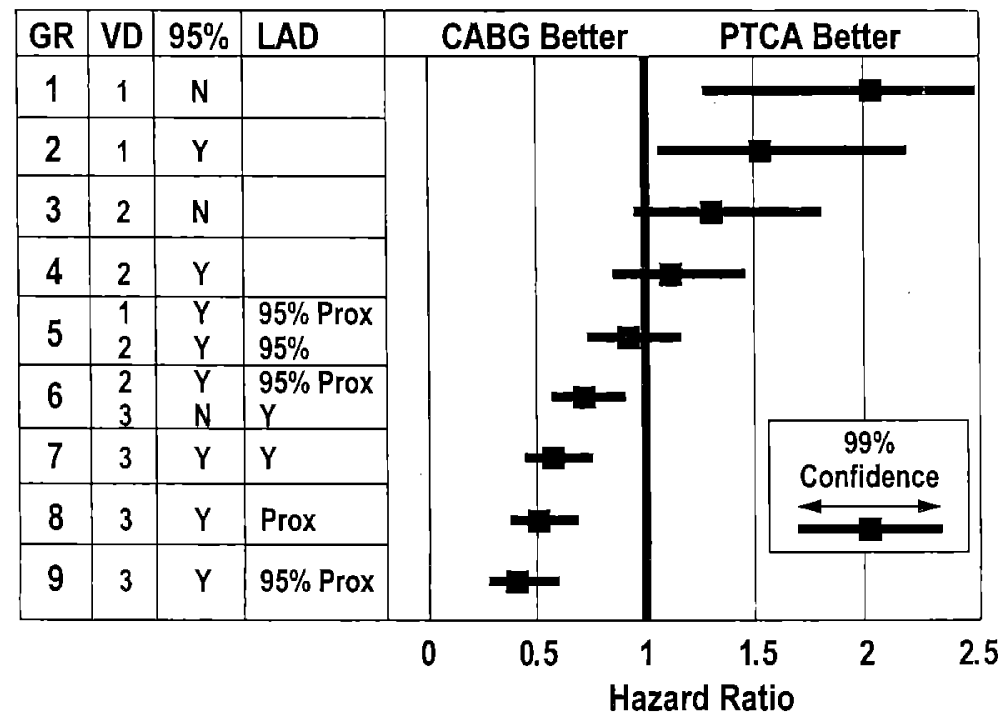

Fig. 9. Adjusted hazard ratios comparing CABG and PTCA for the nine coronary anatomy groups. $V D$, Number of diseased vessels; $95 \%$, $\geq 95 \%$ coronary artery stenosis; Prox, proximal.
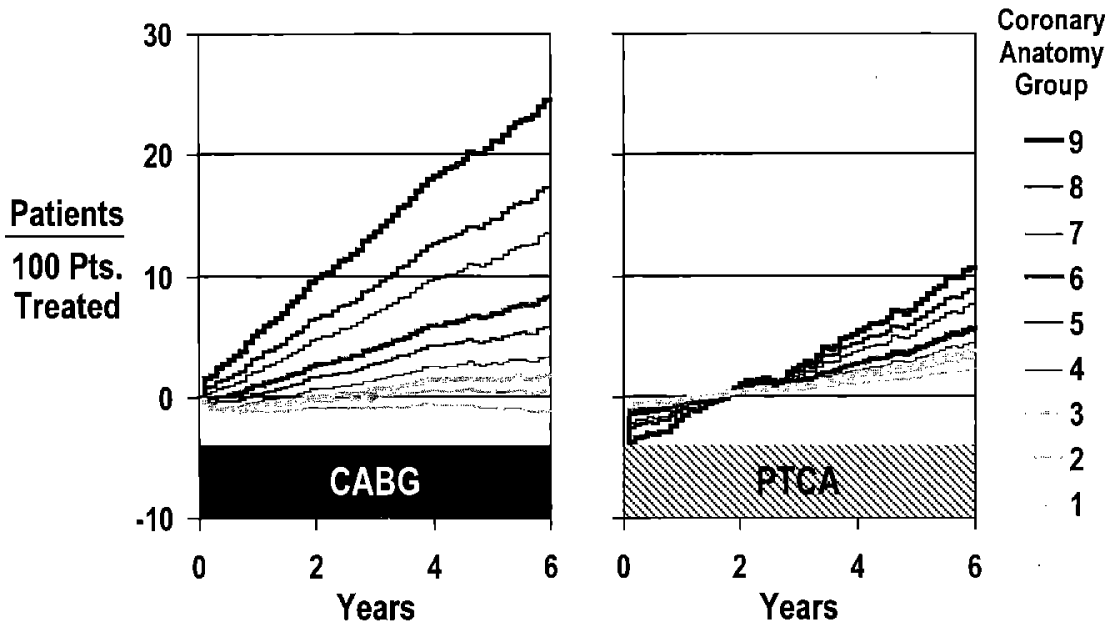

Fig. 10. Absolute survival advantage of CABG and PTCA for each coronary anatomy group expressed as extra patients expected to survive for each 100 patients receiving the interventional treatment instead of medical treatment.

tients in groups 6 through 9 have greater survival when treated with $\mathrm{CABG}$.

In addition to hazard ratios showing relative risk, absolute survival differences expected to result from alternate treatment choices are important indicators of the magnitude of benefit associated with a treatment in a given population. Absolute survival benefit can be expressed as the extra number of patients expected to live if the same patient population were to receive the better of two treatment options. Data depicting the 6-year time course of absolute survival advantage of the two interventional treatments were expressed as the extra numbers of patients within each coronary anatomy group surviving per 100 patients treated with CABG or PTCA compared with survival if these patients had received medical treatment (Fig. 10). The absolute survival advantage increases as a function of CAD severity in both interventional treatment populations and is greatest in patients in coronary anatomy group 9 treated with CABG.

Difference in Kaplan-Meier survival curves be- 


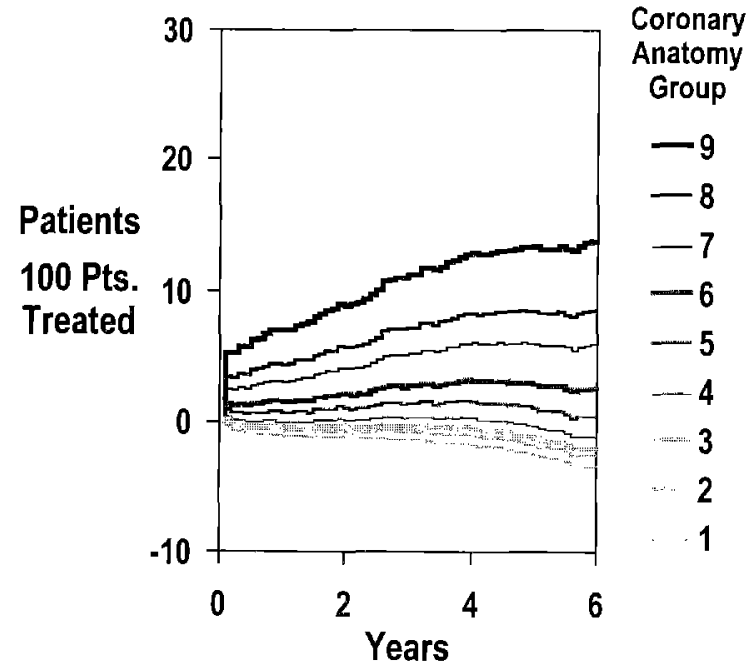

Fig. 11. Absolute survival advantage of CABG for each of the nine anatomic subgroups expressed as extra patients expected to survive for each 100 patients receiving CABG instead of PTCA.

tween CABG and PTCA treatment groups depict the time course over 6 years of the absolute survival advantage of CABG versus PTCA. The positive or negative difference, expressed as extra patients per 100 patients, reflects the average difference in survival outcome in each of the nine coronary anatomy groups expected if all patients had been treated with CABG instead of PTCA (Fig. 11). These data show CABG to have a survival disadvantage for patients with the least severe $\mathrm{CAD}$ and advantage for patients with the most severe CAD versus PTCA. Curves describing differences in survival for the two interventional therapies are not symmetric around zero, and fewer deaths result from use of CABG in the less severe coronary groups than from use of PTCA in patient subgroups with severe CAD. These data predict absolute treatment-related differences in an average patient in each anatomic group, and reasonable clinical judgment must be used in flexibly applying these principles to selection of treatment in an individual case. Other clinical factors known to increase risk, such as severe ischemia, would magnify treatment differences expected for a specific patient.

\section{Discussion}

This study provides unique data on a large series of consecutive patients with one-, two-, and threevessel CAD, permitting evaluation of clinical factors related to survival for patients treated by $\mathrm{CABG}$, PTCA, or medical therapy. The anatomic extent of
CAD was determined to be the dominant variable predictive of treatment-related differences. Categories of information about coronary anatomy related to long-term survival included the number of $75 \%$ and $95 \%$ stenoses and the severity and location of LAD coronary involvement. With these anatomic descriptors, a nine-level coronary anatomy score was found to better define treatment differences than a less descriptive classification of one-, two-, or threevessel disease. Survival at all times decreased in all treatment groups as a function of increasing CAD coronary anatomy score. This relationship was least strong in patients treated with $\mathrm{CABG}$, however, and was strongest in patients treated medically.

Hazard ratios comparing each pair of the three treatments for each of the nine coronary anatomy groups defined clear principles useful in selection of treatment for CAD. Either one or both interventional treatments provided better survival than did medical therapy for all coronary anatomy groups. The magnitudes of absolute survival difference between medical therapy and both interventional therapies were least for the lowest CAD groups, however, and increased progressively through group 9 . This study is the first to conclusively show survival benefit of any interventional therapy over medical therapy in patient groups with the least severe forms of CAD.

No randomized trial has yet compared survival outcomes of PTCA and medical treatment because of the large sample size needed to show benefit in the population with one- or two-vessel CAD, which typically has a low cardiac death rate. The ACME trial is the only randomized trial that has compared PTCA with medical therapy. ${ }^{8}$ This trial, conducted on 212 patients with single-vessel disease, was designed to evaluate the effectiveness of PTCA in relieving myocardial ischemia. In this study, PTCA decreased the incidence of symptomatic ischemia and was associated with more normal results of treadmill examinations.

Yusuf and colleagues ${ }^{9}$ performed a metaanalysis on the outcomes of 2649 patients randomly assigned to receive $\mathrm{CABG}$ or medical management for $\mathrm{CAD}$ in seven individual trials conducted between 1972 and 1984. These data documented the survival benefit of CABG to be greatest for patients with anatomically severe $\mathrm{CAD}$, indexed by number of diseased vessels and the presence of LAD disease. Although there were 1130 patients with one-vessel or two-vessel disease, the risk was low and only 113 deaths overall had occurred at 5 years. A nonsignif- 


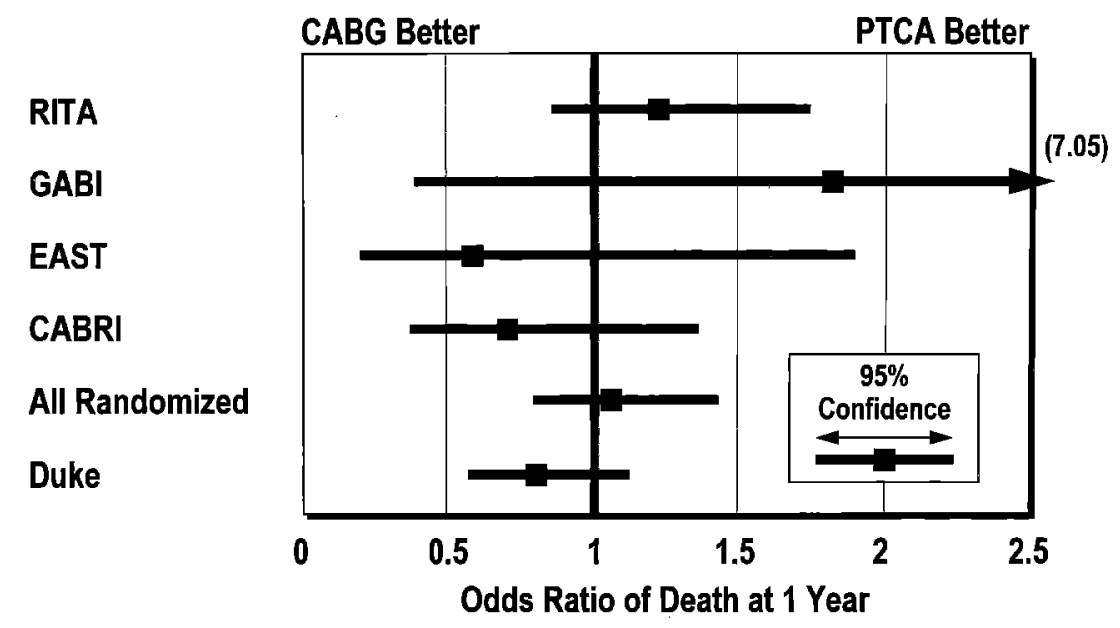

Fig. 12. Mean and 95\% CL hazard ratios of death at 1 year illustrate results of four randomized trials individually and combined to reflect results of 2794 randomly assigned patients. (RITA, ${ }^{10}$ EAST, ${ }^{11}$ GABI, ${ }^{12}$ and CABRI (unpublished data). All Randomized, Metaanalysis of patients in RITA, EAST, GABI, and CABRI; Duke, 3648 patients with two- and three-vessel disease reported on in this study undergoing $\mathrm{CABG}$ or PTCA.

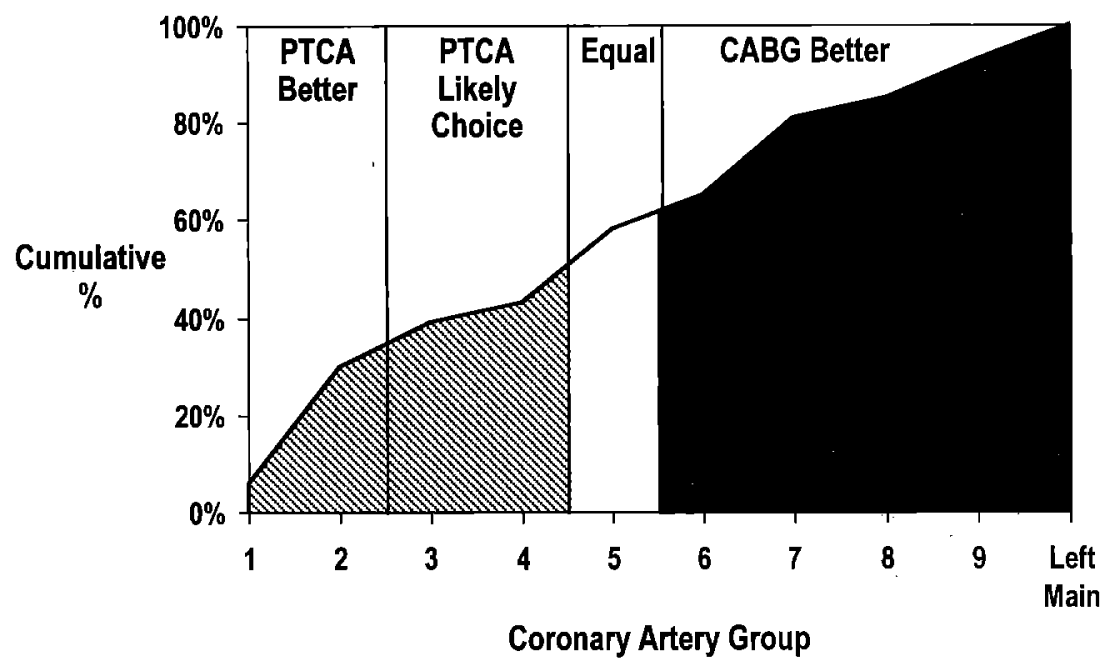

Fig. 13. Cumulative percentages of patients catheterized at Duke during the time of this study with each level of $\mathrm{CAD}$ anatomic severity, including left main stenosis, suggests likely frequency of use of interventional treatments for CAD in catheterized patients.

icant trend toward lower mortality with $\mathrm{CABG}$ was observed (one-vessel odds ratio 0.54 [95\% CL 0.22 to 1.33]; two-vessel odds ratio 0.84 [ $95 \% \mathrm{CL} 0.54$ to 1.32]). Mortality at 5 years was also significantly lower with $\mathrm{CABG}$ among 1341 patients with threevessel disease (odds ratio 0.58 [95\% CL 0.42 0.80 ], $p<0.001$ ). This odds ratio was similar to that determined for the 2771 patients in this study with three-vessel disease ( 0.44 [ $95 \% \mathrm{CL} 0.27$ to 0.74$]$ ).
Comparison of the hazard ratios of patients treated with PTCA and CABG in this study show a clear relationship to the anatomic extent of CAD that favors PTCA in patients with the least severe disease and CABG in patients with the most severe disease. All patients with single-vessel disease, except those with at least $95 \%$ proximal LAD stenosis, benefited from PTCA versus CABG. All patients with three-vessel disease and patients with two- 
vessel disease and at least $95 \%$ proximal $\mathrm{LAD}$ stenosis benefited from CABG versus PTCA. All other patients with two-vessel disease and those with at least $95 \%$ proximal LAD stenosis only had similar survival with either interventional treatment. Absolute survival benefits increased with increasing anatomic severity in both the PTCA and CABG groups, but the magnitude of this increase was greatest with CABG. Additionally, PTCA is associated with a greater need for secondary revascularization procedures than is CABG.

Results have been reported for four randomized trials, three of which have been published, comparing PTCA and CABG for treatment of multivessel CAD. ${ }^{10-12}$ The individual results of these trials and their data combined by metaanalysis fail to show a difference in total mortality between CABG and PTCA treatment for 2794 patients with two- or three-vessel CAD. When survival is compared for a single group including all 3648 patients with two- or three-vessel CAD undergoing $\mathrm{CABG}$ or PTCA in this current study, the resulting odds ratio does not show significant benefit for $\mathrm{CABG}$ and does not differ significantly from that of the combined randomized clinical trial data (Fig. 12). Only when patients with two- and three-vessel $\mathrm{CAD}$ in this current study are more appropriately categorized by coronary anatomy score can differences between PTCA and CABG be appreciated. These previously reported randomized trials did not use a coronary disease index similar to that of the Duke Cardiovascular Database. In the future, it will be useful to compare results from randomized and observational studies to cross-validate results of both methodologies. Results of this study suggest, however, that the "no survival difference between PTCA and CABG for multivessel CAD treatment" conclusion of these trials may change to "benefit from PTCA for some patients with two-vessel CAD and benefit from CABG for most patients with three-vessel CAD." This change in conclusion is likely to require that larger study populations with more detailed descriptions of coronary anatomy be used in analysis of trial results.

To the degree that the results of this study from a single institution can be generalized to other care environments, an expected frequency of use of treatments for CAD can be estimated from the cumulative percentage of patients as a function of CAD severity documented by appropriately indicated coronary angiography (Fig. 13). The cohort of patients considered for intervention must be increased to include patients with left main disease who are known to benefit from coronary bypass. PTCA definitely offers survival benefit to the $35 \%$ of the patient population in coronary anatomy groups 1 and 2. Although PTCA does not offer statistically significant survival benefit to the $15 \%$ of patients in groups 3 and 4 , the trend in survival comparison favors this modality, which also is less invasive than $\mathrm{CABG}$ and therefore is the most likely choice of interventional treatment for patients in these groups. The $10 \%$ of patients in group 5 show no clear benefit for either treatment. The remaining $40 \%$ of catheterized patients with three-vessel and left main disease benefit significantly from CABG.

Data presented in this report provide a conceptual framework to aid selection of treatment for an individual patient with $\mathrm{CAD}$. Many of the treatment differences found to be statistically significant are of borderline clinical significance because of modest differences demonstrated in absolute survival rates. Additionally, this reports focuses only on cardiac survival as an outcome, and many other outcomes validly influence choice of therapy by patients and practitioners. Principles defined by this work are compatible with two clinical practice guidelines developed by national groups from scientific evidence and expert opinion. ${ }^{13}{ }^{14}$ This entire base of knowledge must be flexibly applied according to good clinical judgment to provide the highest quality of care for each individual patient.

This study has several limitations. Treatment was not randomly assigned, and choice of treatment was influenced by factors considered likely to optimize outcome. The validity of comparisons of effectiveness of treatments therefore depends on accurate statistical adjustment for differences in baseline characteristics. Although accuracy of this adjustment cannot be directly assessed, comparison of outcome data from the Duke Cardiovascular Database adjusted by these methodologies has in the past been shown to be comparable to outcome data assessing treatment differences for similar patients studied in randomized clinical trials. ${ }^{15}$ The similarity of outcomes by two comparisons with randomized trial data of results of this study further support the reasonableness of using statistical adjustment to correct observed results for baseline patient differences among treatment groups. Substantial differences among the three treatment groups were observed in important baseline characteristics, however, such 
as anatomic extent of $\mathrm{CAD}$ and incidence of acute myocardial infarction. Available randomized trial data are inadequate to test the validity of our statistical adjustment across this wide range of clinical variables.

Another limitation of the study is definition of treatment groups. The technique of identifying time zero of the interventional groups by the time of initial therapy places all deaths during the waiting period for interventional therapy in the medical cohort. This influence is balanced to some extent by the fact that any subsequent cardiac deaths among patients whose conditions deteriorate with medical treatment and who underwent myocardial revascularization are attributed to the interventional procedure, even though deterioration during medical treatment might have contributed to the ultimate outcome. Because few deaths attributed to the medical cohort occurred early after cardiac catheterization, most such deaths apparently followed the decision to pursue medical therapy as the definitive treatment. The study design used, which permits the strategy of initially pursuing medical therapy with the option to obtain a subsequent interventional procedure, mimics commonly used clinical care strategies. Use of an initial period of medical treatment to evaluate the response of an individual patient before considering interventional therapy was common during this study, and continued use of this strategy is supported by study results, especially for patients with less severe CAD.

A concern inherent in all clinical trials is that improvements in therapy occurring during the interval needed for follow-up may partially invalidate evaluation of the therapy before these improvements. Although CABG has been the most commonly performed cardiac operation for more than two decades, refinements of anesthetic and operative techniques and postoperative care continue to improve outcomes of this procedure. Use of stents is becoming common in PTCA and may enhance the effectiveness of this therapy. In addition, medical therapy now often involves application of aggressive behavior modification to decrease or prevent the risk of untoward events from documented CAD. Prospective compilation of clinical information on a consecutive cohort of well-defined patient populations with CAD and follow-up of outcomes of treatment provide the best approach to evaluate the value of these changes for patients with CAD.

\section{REFERENCES}

1. Favaloro RG. Saphenous vein graft in the surgical treatment of coronary artery disease: operative technique. J Thorac Cardiovasc Surg 1969;58:178-85.

2. Grüntzig AR, Senning A, Siegenthaler WE. Nonoperative dilatation of coronary artery stenosis: percutaneous transluminal coronary angioplasty. N Engl J Med 1979;301:61-8.

3. Mark DB, Nelson CL, Califf RM, et al. Continuing evolution of therapy for coronary artery disease: initial results from the era of coronary angioplasty. Circulation 1994;89:2015-25.

4. Alderman EL, Fisher LD, Litwin P, et al. Results of coronary artery surgery in patients with poor left ventricular function (CASS). Circulation 1983;68:785-95.

5. Chaitman BR, Davis K, Fisher LD, et al. A life table and Cox regression analysis of patients with combined proximal left anterior descending and proximal left circumflex coronary artery disease: non-left main equivalent lesions (CASS). Circulation 1983;68:1163-70.

6. Califf RM, Harrell FE Jr, Lee KL, et al. The evolution of medical and surgical therapy for coronary artery disease: a 15-year perspective. JAMA 1989;261:2077-86.

7. Smith LR, Harrell FE Jr, Rankin JS, et al. Determinants of early versus late cardiac death in patients undergoing coronary artery bypass graft surgery. Circulation 1991;84(Suppl 1):III-245-53.

8. Parisi AF, Folland ED, Hartigan P. A comparison of angioplasty with medical therapy in the treatment of single-vessel coronary artery disease: Veterans Affairs ACME Investigators. N Engl J Med 1992;326:56-8.

9. Yusuf S, Zucker D, Peduzzi P, et al. Effect of coronary artery bypass graft surgery on survival: overview of 10-year results from randomised trials by the Coronary Artery Bypass Graft Surgery Trialists Collaboration. Lancet 1994;344:563-70.

10. RITA Trial Participants. Coronary angioplasty versus coronary artery bypass surgery: the Randomised Intervention Treatment of Angina (RITA) trial. Lancet 1993;341:573-80.

11. King SB 3rd, Lembo NJ, Weintraub WS, et al. A randomized trial comparing coronary angioplasty with coronary bypass surgery. N Engl J Med 1994;331:1044-50.

12. Hamm CW, Reimer J, Ischinger T, Rupprecht H, Berger J, Bleifeld W, German Angioplasty Bypass Surgery Investigation. A randomized study of coronary angioplasty compared with bypass surgery in patients with symptomatic multivessel coronary disease. N Engl J Med 1994;331:1037-43.

13. Kirklin JW, Akins CW, Blackstone EH, et al, for the ACC/ AHA Task Force Report. Guidelines and indications for coronary artery bypass graft surgery: a report of the American College of Cardiology/American Heart Association Task Force on Assessment of Diagnosicic and Therapeutic Cardiovascular Procedures (Subcommittee on Coronary Artery Bypass Graft Surgery). J Am Coll Cardiol 1991;17:543-89.

14. Braunwald E, Mark DB, Jones RH, et al. Unstable angina: diagnosis and management. Clinical practice guideline number 10 (amended), AHCPR publication no 94-0602. Rockville, Maryland: Agency for Health Care Policy and Research, National Heart, Lung, and Blood Institute, Public Health Service, US Department of Health and Human Services, May 1994.

15. Muhlbaier LH, Pryor DB, Rankin JS, et al. Observational comparison of event-free survival with medical and surgical therapy in patients with coronary artery disease: 20 years of follow-up. Circulation 1992;86(Suppl):II198-204. 


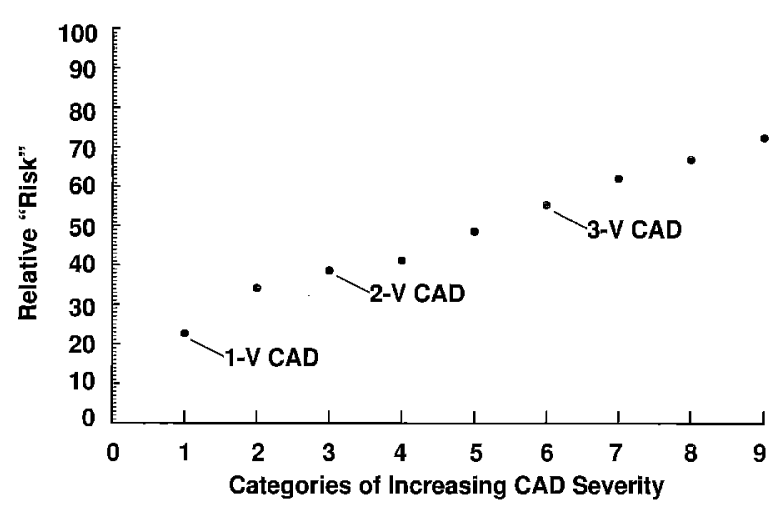

Discussion Fig. 1. Relative "risk" according to severity of disease classification. $1-V$, one-vessel disease; $2-V$, twovessel disease; $3-V$, three-vessel disease.

\section{Discussion}

Dr. John W. Kirklin (Birmingham, Ala.). Dr. Jones, my colleague Gene Blackstone and I congratulate you on an exquisite, masterly, and epochal presentation. We are not casual observers of your work, but rather are intense students of it. Our own work during this period has emphasized the time-related hazard function rather than the hazard ratio and patient-specific predictions of outcomes after alternative forms of therapy rather than average values. These are all details, however, that when well understood lead to similar conclusions. These somewhat parallel courses have given us a high respect for your work.

You and your colleagues have today undertaken a Herculean task, the task of presenting to us in understandable form highly relevant information generated by at least 10 years of clinical and statistical work by one of the most talented and experienced groups in the world, headed by you and Dr. Frank Harrell.

Dr. Jones has presented this information in a natural language, English. In contrast, the work has been done, and could only have been done, in the language of mathematics. He has thus been required to present to us a translation from mathematics to the English language, and in such translations at least a little is always lost.

The only contribution we can make to Dr. Jones' masterly presentation comes from the friendly and respectful competition they have allowed us to develop with them, which has led to our ability to translate back and forth, so to speak. As Dr. Jones has told you, this work has been done in the domains of Cox hazard ratios and of average values (Discussion Fig. 1). As described in detail in the paper itself, which Dr. Jones was kind enough to send us a few weeks ago, these categories are not strictly those of the extent of the CAD. Rather, the number, severity, and location of the stenoses contribute to the categorization as well, as you probably noted on the slides. The dot between one-vessel and twovessel disease is thus neither of these categories, but is in fact an intermediate one between the two. There are two categories between two-vessel and three-vessel

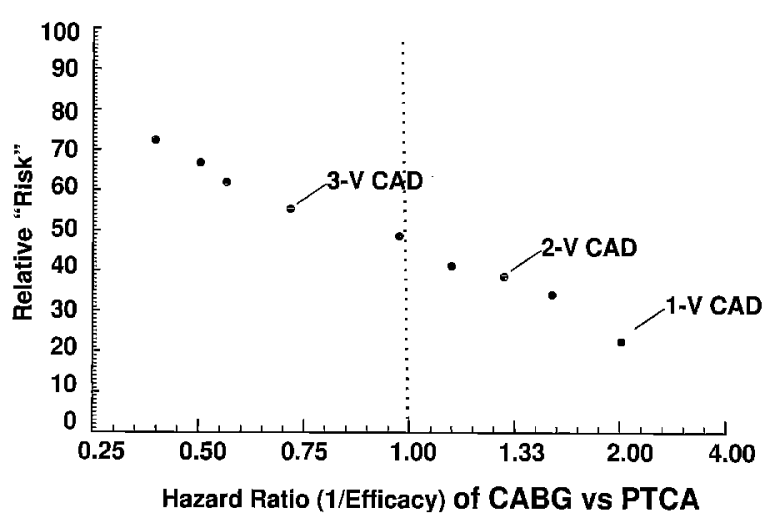

Discussion Fig. 2. Hazard ratio versus relative "risk." $1-V$, one-vessel disease; $2-V$, two-vessel disease; $3-V$, three-vessel disease.

CAD, and so forth. You may recall from the slides that a $95 \%$ left main stenosis was associated with a relative risk of 100. "Risk" is in quotes because it is in a way shorthand for a more complicated expression. Dr. Jones has already emphasized, very appropriately in our judgment, that the more severe the CAD category, the greater the so-called risk imposed by the disease. For this concept, he has used "relative prognostic weight." An enormous amount of work lies behind this relatively simple depiction.

This (Discussion Fig. 2) is conceptually identical to Dr. Jones' Fig. 9, and I show this simplified version only to underline the importance of his message. Dr. Jones and colleagues work and think in terms of the hazard ratio. Ratios less than 1.0 indicate increasing effectiveness or efficiency of CABG over PTCA and ratios greater than 1.0 depict lessened efficacy. In other words, the hazard ratio is the inverse of efficacy. This again states the point that Dr. Jones and colleagues have discovered, namely that the effectiveness of CABG over PTCA gets greater, as shown by the decreasing hazard ratio, as the severity of $C A D$ increases.

Dr. Smith. I believe that your use of our data points out one of the advantages that we need to enjoy more, which is sharing of the individual patient individual data so that they can be compared by multiple methods. It is difficult to compare our data with other data, and as you see, we have presented $\mathrm{CAD}$ as more of a continuous variable than the usual classification as one-, two-, and three-vessel disease. Access to the raw data and sharing between institutions are important features to advance our understanding of coronary disease.

Although these data support the concept that revascularization improves relative survival for all patients with significant-that is, greater than $75 \%$ stenosis - coronary disease, the absolute magnitude of the survival benefit is greatest for patients with three-vessel disease or two-vessel disease with 95\% proximal LAD stenosis. Other attributes known to affect surgical risk and enhance surgical outcome must be considered when evaluating individual patients within this framework. The ultimate indications for PTCA and CABG 
will also depend on symptomatic relief, freedom from reintervention, cost, and economic benefit. Reduction of CABG cost, effective rehabilitation after $\mathrm{CABG}$, and reduction in mortality and morbid complications continue to be major goals for thoracic surgeons.

\section{Appendix 1*}

\section{Survival equation:}

$$
\mathrm{S}_{t}=\mathrm{S}_{\mathrm{o}}(t)^{\exp (\mathrm{x})}
$$

The probability of being alive at any time $S_{(t)}$ is equal to the underlying population survival curve $S_{0}$ at time $t$ raised to the $\mathrm{e}^{\mathrm{x}}$ power, where

$$
\begin{aligned}
& e=\text { base of the natural logarithm } \\
& x=a_{1} y_{1}+a_{2} y_{2}+\cdots+a_{l} y_{k}
\end{aligned}
$$

*The appendix has been provided at the request of the Editor. This appendix should not be used by other institutions that might define these variables differently or whose treatment approaches might differ from those of the authors and influence the relative weight of these variables. The interested reader may obtain a copy of the variance-covariance matrix from the authors. where $y_{1}, y_{2}, \cdots \cdot$, and $y_{k}$ are the characteristics and $a_{1}$, $a_{2}, \cdot \cdot$, and $a_{k}$ are the corresponding Cox proportional hazard regression coefficients.

The predictive characteristics are listed in Appendix Table 1 with their coefficients.

Appendix Table 1. Cox proportional hazards model

\begin{tabular}{lcc}
\hline & Coefficient & $\begin{array}{c}S E \\
\text { (coefficient) }\end{array}$ \\
\hline CAD index & 0.02896 & 0.00280 \\
Age & 0.04022 & 0.00270 \\
EF (truncated at 0.60 ) & -0.04708 & 0.00224 \\
Mitral insufficiency & 0.13173 & 0.03633 \\
Gender & 0.09712 & 0.05594 \\
Vascular disease index & 0.32631 & 0.03599 \\
CHF (yes/no) & 0.59149 & 0.06079 \\
Comorbidity & 0.51628 & 0.07021 \\
MI/unstable angina index & -0.14405 & 0.01529 \\
Chest pain (yes/no) & -0.14113 & 0.06652 \\
PTCA/CAD index & 0.00213 & 0.00544 \\
CABG/CAD index & -0.02904 & 0.00465 \\
Centering constant & 0.6068266 &
\end{tabular}

$C A D$, Coronary artery disease; $E F$, ejection fraction; $C H F$, congestive heart failure; $M I$. myocardial infarction; $P T C A$, percutaneous transluminal coronary angioplasty; $C A B G$, coronary artery bypass grafting; $S E$, standard error. 